\title{
On constructing differential equations with singularities from incomplete spectral information
}

\author{
G. Freiling, Fachbereich Mathematik, \\ Universität Duisburg, \\ D-47048 Duisburg, Germany, \\ Email: freiling@math.uni-duisburg.de \\ V. Yurko, Department of Mathematics, \\ Saratov University, \\ 410600 Saratov, Russia, \\ Email: Yurko@scnit.saratov.su
}

\begin{abstract}
We study the inverse problem of synthesizing parameters of differential equations with singularities from incomplete spectral information. We establish properties of the spectral characteristics, obtain conditions for the solvability of such classes of inverse problems and provide algorithms for constructing the solution.
\end{abstract}

AMS classification: 34A55, 34B05, 34E20, 34L05, 47E05

\section{Introduction.}

Let us consider the system

$$
\frac{d y_{1}}{d x}=i \rho R(x) y_{2}, \frac{d y_{2}}{d x}=i \rho \frac{1}{R(x)} y_{1}, x \in[0, T]
$$

with the initial conditions

$$
y_{1}(0, \rho)=1, y_{2}(0, \rho)=-1
$$

Here $\rho=k+i \tau$ is the spectral parameter, and $R(x)$ is a real function.

For a wide class of problems describing the propagation of electromagnetic waves in a stratified medium, Maxwell's equations can be reduced to the canonical form (1), where $x$ is the variable in the direction of stratification, $y_{1}$ and $y_{2}$ are the components of the electromagnetic field, $R(x)$ is the wave resistance which describes the refractive properties of the medium and $\rho$ is the wave number in a vacuum [1, 2]. System (1) also appears in radio engineering for the design of directional couplers for heterogeneous 
electronic lines, which constitute one of the important classes of radiophysical synthesis problems [3].

Some aspects of synthesis problems for system (1) with a positive $R(x)$ were studied in [2-4] and other works. In this paper we study the inverse problem for system (1) from incomplete spectral information in the case when $R(x)$ is nonnegative and can has zeros which are called turning points. More precisely, we shall consider two classes of functions $R(x)$. We shall say that $R(x) \in B_{0}$ if $R(x), R^{\prime}(x)$ are absolutely continuous on $[0, T]$, $R^{\prime \prime}(x) \in L_{2}(0, T), R(x)>0, R(0)=1, R^{\prime}(0)=0$. We also consider the more general case when $R(x)$ has zeros $0<x_{1}<\ldots<x_{p}<T, p \geq 0$ inside the interval $(0, T)$. We shall say that $R(x) \in B_{0}^{+}$if $R(x)$ has the form

$$
\frac{1}{R(x)}=\sum_{j=1}^{p} \frac{R_{j}}{\left(x-x_{j}\right)^{2}}+R_{0}(x), 0<x_{1}<\ldots<x_{p}<T, R_{j}>0
$$

and $R_{0}(x), R_{0}^{\prime}(x)$ are absolutely continuous on $[0, T], R_{0}^{\prime \prime}(x) \in L_{2}(0, T), R(x)>0(x \neq$ $\left.x_{j}\right), R(0)=1, R^{\prime}(0)=0$. In particular, if here $p=0$ then $R(x) \in B_{0}$.

As the main spectral characteristics we introduce the amplitude reflection coefficient

$$
r(\rho)=\frac{y_{1}(T, \rho)+R^{0} y_{2}(T, \rho)}{y_{1}(T, \rho)-R^{0} y_{2}(T, \rho)}, \quad R^{0}:=R(T) ;
$$

the power reflection coefficient

$$
\sigma(k)=|r(k)|^{2}, k:=\operatorname{Re} \rho
$$

the transmission coefficients

$$
f_{1}(\rho)=\frac{y_{1}(T, \rho)-R^{0} y_{2}(T, \rho)}{2 \sqrt{R^{0}}}, f_{2}(\rho)=\frac{y_{1}(T, \rho)+R^{0} y_{2}(T, \rho)}{2 \sqrt{R^{0}}}
$$

and the characteristic function

$$
\triangle(\rho)=\frac{1}{\sqrt{R^{0}}} y_{1}(T, \rho)
$$

Clearly,

$$
\begin{gathered}
r(\rho)=f_{2}(\rho) / f_{1}(\rho), \\
\triangle(\rho)=f_{1}(\rho)+f_{2}(\rho) .
\end{gathered}
$$

In many cases of practical interest, the phase is difficult or impossible to measure, while the amplitude is easily accessible to measurement. Such cases lead us to the so-called incomplete inverse problems where only a part of the spectral information is available. In this paper we study one of the incomplete inverse problems, namely, the inverse problem of recovering the wave resistance from the power reflection coefficient:

Inverse Problem 1. Given $\sigma(k)$, construct $R(x)$.

The lack of spectral information leads here to nonuniqueness of the solution of the inverse problem. Let us briefly describe a scheme of the solution of Inverse Problem 1.

Denote $\alpha_{j}(k)=\left|f_{j}(k)\right|, j=1,2$. Since $\alpha_{1}^{2}(k)-\alpha_{2}^{2}(k) \equiv 1$ (see (19) below), we get in view of $(2)$,

$$
\sigma(k)=1-\left(\alpha_{1}(k)\right)^{-2}, 0 \leq \sigma(k)<1 .
$$


Firstly, from the given power reflection coefficient $\sigma(k)$ we construct $\alpha_{j}(k)$. Then, using analytic properties of the transmission coefficients $f_{j}(\rho)$ and, in particular, information about their zeros, we reconstruct the transmission coefficients from their amplitudes. Namely on this stage we are faced with nonuniqueness. Problems of reconstruction of analytic functions from their moduli often appear in applications and have been studied in many works (see $[5-7]$ and references therein). The last of our steps is to calculate the characteristic function $\triangle(\rho)$ by $(3)$ and to solve the inverse problem of recovering $R(x)$ from $\triangle(\rho)$. Here we use the inverse Sturm-Liouville theory which was developed in [8-12] and other works.

To realize this scheme, in Section 2 we study properties of the spectral characteristics. In Section 3 we solve the synthesis problem for $R(x)$ from the characteristic function $\triangle(\rho)$, obtain necessary and sufficient conditions for its solvability and provide three algorithms for constructing the solution. In Section 4 we study the problem of recovering the transmission coefficients from their moduli. In Section 5, the so-called symmetrical case is considered, and in Section 6 we provide an algorithm for the solution of Inverse Problem 1. We note that inverse problems for Sturm-Liouville equations with turning points have been studied in [13]. Some aspects of the turning point theory and a number of its applications are described in [14 - 16].

We shall say that $R(x) \in B_{0}^{-}$if $(R(x))^{-1} \in B_{0}^{+}$. An investigation of the classes $B_{0}^{+}$and $B_{0}^{-}$is completely similar because the replacement $R \rightarrow 1 / R$ is equivalent to the replacement $\left(y_{1}, y_{2}\right) \rightarrow\left(-y_{2},-y_{1}\right)$. Below it will be more convenient for us to consider the case $R(x) \in B_{0}^{-}$.

\section{Properties of the spectral characteristics.}

We transform (1) by means of the replacement

$$
y_{1}(x, \rho)=\sqrt{R(x)} u(x, \rho), \quad y_{2}(x, \rho)=\frac{1}{\sqrt{R(x)}} v(x, \rho)
$$

to the system

$$
u^{\prime}+h(x) u=i \rho v, v^{\prime}-h(x) v=i \rho u, x \in[0, T]
$$

with the initial conditions $u(0, \rho)=1, v(o, \rho)=-1$, where

$$
h(x)=\frac{R^{\prime}(x)}{2 R(x)} .
$$

Hence the function $u(x, \rho)$ satisfies the equation

$$
-u^{\prime \prime}+q(x) u=\lambda u, \quad \lambda=\rho^{2}
$$

and the initial conditions

$$
u(0, \rho)=1, u^{\prime}(0, \rho)=-i \rho
$$

where

$$
q(x)=h^{2}(x)-h^{\prime}(x)
$$

or

$$
q(x)=\frac{3}{4}\left(\frac{R^{\prime}(x)}{R(x)}\right)^{2}-\frac{1}{2} \frac{R^{\prime \prime}(x)}{R(x)} .
$$


Similarly,

$$
-v^{\prime \prime}+g(x) v=\lambda v, v(0, \rho)=-1, v^{\prime}(0, \rho)=i \rho,
$$

where $g(x)=h^{2}(x)+h^{\prime}(x)$.

In view of (4), the transmission coefficients, the amplitude reflection coefficient and the characteristic function take the form

$$
\begin{array}{ll}
f_{1}(\rho)=\frac{u(T, \rho)-v(T, \rho)}{2}, & f_{2}(\rho)=\frac{u(T, \rho)+v(T, \rho)}{2}, \\
r(\rho)=\frac{u(T, \rho)+v(T, \rho)}{u(T, \rho)-v(T, \rho)}=\frac{f_{2}(\rho)}{f_{1}(\rho)}, & \triangle(\rho)=u(T, \rho) .
\end{array}
$$

Since $y_{1}(x, 0) \equiv 1$, we have according to (4)

$$
\sqrt{R(x)} u(x, 0) \equiv 1 \text {. }
$$

Lemma 1. $R(x) \in B_{0}^{-}$if and only if $q(x) \in L_{2}(0, T), \triangle(0) \neq 0$.

Proof. 1) Let $R(x) \in B_{0}^{-}$, i.e.

$$
\left.\begin{array}{l}
R(x)=\sum_{j=1}^{p} \frac{R_{j}}{\left(x-x_{j}\right)^{2}}+R_{0}(x), \quad 0<x_{1}<\ldots<x_{p}<T, \quad R_{j}>0, \\
R_{0}(x) \in W_{2}^{2}(0, T), R(x)>0\left(x \neq x_{j}\right), R(0)=1, R^{\prime}(0)=0 .
\end{array}\right\}
$$

Using (6) and (11) we get for $x \rightarrow x_{j}$

$$
h(x) \sim-\frac{1}{x-x_{j}}+h_{j}^{*}(x), \quad h_{j}^{*}(x) \in W_{2}^{1}, \quad h_{j}^{*}\left(x_{j}\right)=0,
$$

and consequently $q(x) \in L_{2}(0, T)$. If follows from $(10)$ that $u(T, 0) \neq 0$, i.e. $\triangle(0) \neq 0$.

2) Let now $q(x) \in L_{2}(0, T), u(T, 0) \neq 0$, and let $0<x_{1}<\ldots<x_{p}<T$ be zeros of $u(x, 0)$. If follows from (10) that

$$
R(x)=(u(x, 0))^{-2} .
$$

Hence $R(x)>0\left(x \neq x_{j}\right), R(0)=1, R^{\prime}(0)=0$, and

$$
R(x) \sim \frac{R_{j}}{\left(x-x_{j}\right)^{2}}, x \rightarrow x_{j}, R_{j}>0 .
$$

Denote

$$
R_{0}(x):=R(x)-\sum_{j=1}^{p} \frac{R_{j}}{\left(x-x_{j}\right)^{2}} .
$$

It is easy to see that $R_{0}(x) \in W_{2}^{2}[0, T]$. Lemma 1 is proved.

Remark 1. If $R(x) \in B_{0}^{-}$, then the functions $y_{1}(x, \rho)$ and $v(x, \rho)$ have singularities of order 1 at $x=x_{j}$, and there exist finite limits $\lim _{x \rightarrow x_{j}}\left(x-x_{j}\right) v(x, \rho), \lim _{x \rightarrow x_{j}}\left(x-x_{j}\right) y_{1}(x, \rho)$. In the other words, we continue solutions in the neighbourhoods of the singular points 
with the help of generalized Bessel type solutions (see, [17]). If follows from (10) that $\lim _{x \rightarrow x_{j}}\left(x-x_{j}\right) \sqrt{R(x)}=R_{j}$.

Example. Let $R(x)=\frac{1}{\cos ^{2} x}$. Then $h(x)=\operatorname{tg} x, \quad q(x)=-1, \quad u(x, 0)=\frac{1}{\sqrt{R(x)}}=$ $\cos x, \quad u(x, \rho)=\cos \mu x-i \rho \frac{\sin \mu x}{\mu}, \quad \mu^{2}=\rho^{2}+1$. If $T \in\left(\frac{(2 p-1) \pi}{2}, \frac{(2 p+1) \pi}{2}\right)$, then $u(x, 0)$ has $p$ zeros $x_{j}=\frac{(2 j-1) \pi}{2}, j=\overline{1, p}$.

Theorem 1. Let $R(x) \in B_{0}^{-}$. Then

(i) The characteristic function $\triangle(\rho)$ is entire in $\rho$, and the following representation holds

$$
\triangle(\rho)=e^{-i \rho T}+\int_{-T}^{T} \eta(t) e^{-i \rho t} d t, \eta(t) \in A C[-T, T], \eta^{\prime}(t) \in L_{2}(-T, T), \eta(-T)=0,
$$

where $\eta(t)$ is a real function, and

$$
\eta(T)=\frac{1}{2} \int_{0}^{T} q(t) d t=-\frac{h(T)}{2}+\frac{1}{2} \int_{0}^{T} h^{2}(t) d t .
$$

(ii) For real $\rho, \triangle(\rho)$ has no zeros. For $\operatorname{Im} \rho>0, \triangle(\rho)$ has at most a finite number of simple zeros of the form $\rho_{j}=i \tau_{j}, \tau_{j}>0, j=\overline{1, m}, m \geq 0$.

(iii) The function $\triangle(\rho)$ has no zeros for $\operatorname{Im} \rho \geq 0$ if and only if $R(x) \in B_{0}$.

Proof: 1) It is well-known (see [9]) that $u(x, \rho)$ has the form

$$
u(x, \rho)=e^{-i \rho x}+\int_{-x}^{x} K(x, t) e^{-i \rho t} d t,
$$

where $K(x, t)$ is a real, absolutely continuous function, $K_{t}(T, t) \in L_{2}(-T, T), K(x,-x)=$ $0, K(x, x)=\frac{1}{2} \int_{0}^{x} q(t) d t$. Moreover,

$$
\begin{gathered}
\overline{u(x, \rho)}=u(x,-\bar{\rho}), \\
\langle u(x, \rho), u(x,-\rho)\rangle \equiv 2 i \rho,
\end{gathered}
$$

where $\langle y, z\rangle:=y z^{\prime}-y^{\prime} z$. Hence we arrive at $(12)$, where $\eta(t)=K(T, t)$.

2) If follows from (13) that for real $\rho, \overline{\triangle(\rho)}=\triangle(-\rho)$, and consequently, in view of (14), for real $\rho \neq 0, \triangle(\rho)$ has no zeros. By virtue of Lemma $1, \triangle(0) \neq 0$.

Further, denote $\Pi_{+}=\{\rho: \operatorname{Im} \rho>0\}$. Let $\rho=k+i \tau \in \Pi_{+}$be a zero of $\triangle(\rho)$. Since

$$
-u^{\prime \prime}+q(x) u=\rho^{2} u, \quad-\bar{u}^{\prime \prime}+q(x) \bar{u}=\bar{\rho}^{2} \bar{u},
$$

we get

$$
\left(\rho^{2}-\bar{\rho}^{2}\right) \int_{0}^{T}|u|^{2} d x=\left.\right|_{0} ^{T}\langle u, \bar{u}\rangle=-i(\rho+\bar{\rho}),
$$

which is possible only if $k=0$. Hence, all zeros of $\triangle(\rho)$ in $\Pi_{+}$are pure imaginary, and by virtue of (12), the number of zeros in $\bar{\Pi}_{+}$is finite. 
3) Let us show that in $\Pi_{+}$all zeros of $\triangle(\rho)$ are simple. Suppose that for a certain $\rho=i \tau, \tau>0, \triangle(\rho)=\dot{\triangle}(\rho)=0$, where $\dot{\triangle}(\rho)=\frac{d}{d \rho} \triangle(\rho)$. Denote $u_{1}=\dot{u}$. Then, by virtue of $(7)$,

$$
-u_{1}^{\prime \prime}+q(x) u_{1}=\rho^{2} u_{1}+2 \rho u, u_{1}(0, \rho)=0, u_{1}^{\prime}(0, \rho)=-i .
$$

Consequently,

$$
\begin{gathered}
2 \rho \int_{0}^{T} u^{2}(x, \rho) d x=\int_{0}^{T} u(x, \rho)\left(-u_{1}^{\prime \prime}(x, \rho)+q(x) u_{1}(x, \rho)-\rho^{2} u_{1}(x, \rho)\right) d x= \\
-\left.\right|_{0} ^{T}\left\langle u, u_{1}\right\rangle=-i
\end{gathered}
$$

i.e.

$$
2 \tau \int_{0}^{\tau}|u(x, \rho)|^{2} d x=-1
$$

which is impossible.

4) If $R(x) \in B_{0}$, then in view of (10) $u(x, 0)>0, x \in[0, T]$. Since $u(0, i \tau)=1, \tau \geq 0$ and $u(x, i \tau)>0, x \in[0, T]$ for large $\tau$, we get $u(T, i \tau)>0$ for all $\tau \geq 0$, i.e. $\triangle \rho)$ has no zeros in $\bar{\Pi}_{+}$. The inverse assertion is proved similarly. Theorem 1 is proved.

In the analogous manner one can prove the following theorem.

Theorem 2. Let $R(x) \in B_{0}^{-}$.

(i) The functions $f_{1}(\rho), f_{2}(\rho)$ are entire in $\rho$, and has the form

$$
\begin{gathered}
f_{1}(\rho)=e^{-i \rho T}+\int_{-T}^{T} g_{1}(t) e^{-i \rho t} d t, g_{1}(t) \in A C[-T, T], g_{1}^{\prime}(t) \in L_{2}(-T, T), g_{1}(-T)=0 \\
f_{2}(\rho)=\int_{-T}^{T} g_{2}(t) e^{-i \rho t} d t, g_{2}(t) \in A C[-T, T], g_{2}^{\prime}(t) \in L_{2}(-T, T), g_{2}(-T)=0,
\end{gathered}
$$

where $g_{j}(t)$ are real, and

$$
g_{1}(T)=\frac{1}{2} \int_{0}^{T} h^{2}(t), g_{2}(T)=-\frac{h}{2}, h:=h(T) .
$$

(ii)

$$
f_{1}(\rho) f_{1}(-\rho)-f_{2}(\rho) f_{2}(-\rho) \equiv 1 \text {. }
$$

(iii) For real $\rho, f_{1}(\rho)$ has no zeros. In $\bar{\Pi}_{+}, f_{1}(\rho)$ has a finite number of simple zeros of the form $\rho_{j}^{*}=i \tau_{j}^{*}, \tau_{j}^{*}>0, \quad j=\overline{1, m^{*}}, \quad m^{*} \geq 0$.

(iv) If $R(x) \in B_{0}$, then $f_{1}(\rho)$ has no zeros in $\bar{\Pi}_{+}$, i.e. $m^{*}=0$.

We note that (18) follows from (14). Since $\overline{f_{j}(\rho)}=f_{j}(-\bar{\rho})$, we obtain from (18) that

$$
\alpha_{1}^{2}(k)-\alpha_{2}^{2}(k) \equiv 1 \text {. }
$$


It follows from (9), (15), (16) and (19) that

$$
\left.\begin{array}{l}
\alpha_{1}^{2}(k)=1+\frac{h^{2}}{4 k^{2}}+\frac{\omega(k)}{k^{2}}, \quad \alpha_{2}^{2}(k)=\frac{h^{2}}{4 k^{2}}+\frac{\omega(k)}{k^{2}}, \\
\sigma(k)=\frac{h^{2}}{4 k^{2}+h^{2}}+\frac{\omega_{1}(k)}{k^{2}},|k| \rightarrow \infty,
\end{array}\right\}
$$

where $\omega(k), \omega_{1}(k) \in L_{2}(-\infty, \infty)$.

Lemma 2. Denote $g_{j}^{*}(t)=g_{j}(t-T)$. Then

$$
g_{1}^{*}(\xi)+\int_{0}^{\xi} g_{1}^{*}(t) g_{1}^{*}(t+2 T-\xi) d t=\int_{0}^{\xi} g_{2}^{*}(t) g_{2}^{*}(t+2 T-\xi) d t, \quad \xi \in[0,2 T] .
$$

Proof. Indeed using (15) and (16) we calculate

$$
f_{1}(\rho) f_{1}(-\rho)=1+\int_{-2 T}^{2 T} G_{1}(\xi) e^{-i \rho \xi} d \xi, \quad f_{2}(\rho) f_{2}(-\rho)=\int_{-2 T}^{2 T} G_{2}(\xi) e^{-i \rho \xi} d \xi,
$$

where $G_{j}(-\xi)=G_{j}(\xi)$,

$$
G_{1}(\xi)=g_{1}(T-\xi)+\int_{\xi-T}^{T} g_{1}(t) g_{1}(t-\xi) d t, G_{2}(\xi)=\int_{\xi-T}^{\xi} g_{2}(t) g_{2}(t-\xi) d t, \quad \xi>0 .
$$

Substituting (22) into (18) we arrive at (21).

Denote

$$
p(x)=\left\{\begin{array}{cc}
q(T-x) & , \quad 0 \leq x \leq T \\
0 & , \quad x>T
\end{array}\right.
$$

and consider the equation

$$
-y^{\prime \prime}+p(x) y=\lambda y, x>0
$$

on the half-line. Let $e(x, \rho)$ be the Iost solution of $(23)$ such that $e(x, \rho) \equiv e^{i \rho x}$ for $x \geq T$. Denote $\delta(\rho)=e(0, \rho)$. Clearly,

$$
e(x, \rho)=e^{i \rho T} u(T-x, \rho), 0 \leq x \leq T, \quad \delta(\rho)=e^{i \rho T} \triangle(\rho) .
$$

Then

$$
\begin{gathered}
e(x, \rho)=e^{i \rho x}+\int_{x}^{2 T-x} G(x, t) e^{i \rho t} d t, \quad 0 \leq x \leq T ; \quad \epsilon(x, \rho) \equiv e^{i \rho x}, \quad x \geq T, \\
\delta(\rho)=1+\int_{0}^{2 T} \theta(t) e^{i \rho t} d t, \quad \theta(t) \in A C[0,2 T], \quad \theta^{\prime}(t) \in L_{2}(0,2 T), \quad \theta(2 T)=0,
\end{gathered}
$$

where $G(x, t)=K(T-x, T-t), \quad \theta(t)=\eta(T-t)=G(0, t), \quad G(x, 2 T-x)=0$, $\theta(0)=\frac{1}{2} \int_{0}^{T} p(t) d t, \quad G(x, x)=\frac{1}{2} \int_{x}^{T} p(\xi) d \xi$.

Denote

$$
\alpha_{j}=\left(\int_{0}^{\infty} e^{2}\left(x, \rho_{j}\right) d x\right)^{-1}>0, \rho_{j}=i \tau_{j}, \quad \tau_{j}>0, j=\overline{1, m} .
$$

\section{Lemma 3.}

$$
\alpha_{j}=\frac{i \delta\left(-\rho_{j}\right)}{\dot{\delta}\left(\rho_{j}\right)}
$$


where $\dot{\delta}(\rho)=\frac{d}{d \rho} \delta(\rho)$

Proof. Since $-e^{\prime \prime}+p(x) e=\rho^{2} e$ and $\delta\left(\rho_{j}\right)=0$, we have

$$
\left(\rho^{2}-\rho_{j}^{2}\right) \int_{0}^{\infty} e(x, \rho) e\left(x, \rho_{j}\right) d x=\left.\right|_{0} ^{\infty}\left\langle e(x, \rho), e\left(x, \rho_{j}\right)\right\rangle=-e^{\prime}\left(0, \rho_{j}\right) \delta(\rho) .
$$

If yields

$$
\alpha_{j}=-\frac{2 \rho_{j}}{e^{\prime}\left(0, \rho_{j}\right) \dot{\delta}\left(\rho_{j}\right)} .
$$

Since $\langle e(x, \rho), e(x,-\rho)\rangle \equiv 2 i \rho$, we get

$$
\delta\left(-\rho_{j}\right) \epsilon^{\prime}\left(0, \rho_{j}\right)=2 i \rho_{j} .
$$

Together with (27) it gives (26).

\section{Synthesis of $R(x)$ from the characteristic function $\triangle(\rho)$.}

In this section we study the inverse problem of recovering the wave resistance $R(x)$ from the given $\triangle(\rho)$ in the class $B_{0}^{-}$. This inverse problem has a unique solution. We provide physical realization conditions, i.e. necessary and sufficient conditions on a function $\triangle(\rho)$ to be the characteristic function for a certain $R(x) \in B_{0}^{-}$. We also obtain three algorithms for the solution of the inverse problem.

Denote $s(\rho)=\frac{\delta(-\rho)}{\delta(\rho)}$. For real $\rho, s(\rho)$ is continuous and, by virtue of $(25), s(\rho)=$ $1+O\left(\frac{1}{\rho}\right),|\rho| \rightarrow \infty$. Consequently, $1-s(\rho) \in L_{2}(-\infty, \infty)$. We introduce the Fourier transform

$$
\begin{gathered}
F_{0}(t)=\frac{1}{2 \pi} \int_{-\infty}^{\infty}(1-s(\rho)) e^{i \rho t} d \rho, \\
1-s(\rho)=\int_{-\infty}^{\infty} F_{0}(t) e^{-i \rho t} d t .
\end{gathered}
$$

Substituting (25) and (30) into the relation $\delta(\rho) s(\rho)=\delta(-\rho)$, we obtain the connection between $F_{0}(t)$ and $\theta(t)$ :

$$
\theta(t)+F_{0}(t)+\int_{0}^{2 T} \theta(s) F_{0}(t+s) d s=0, \quad t>0
$$

where $\theta(t) \equiv 0$ for $t>2 T$. Further, we consider the function

$$
F(t)=F_{0}(t)+F_{1}(t),
$$

where

$$
F_{1}(t)=\sum_{j=1}^{m} \alpha_{j} e^{i \rho_{j} t}
$$

Since

$$
F_{1}(t)+\int_{0}^{2 T} \theta(s) F_{1}(t+s) d s=\sum_{j=1}^{m} \alpha_{j} e^{i \rho_{j} t} \delta\left(\rho_{j}\right)=0,
$$

we get by virtue of (31) that

$$
\theta(t)+F(t)+\int_{0}^{2 T} \theta(s) F(t+s) d s=0, \quad t>0 .
$$


Calculating integral (29) by Jordan lemma for $t>2 T$ and using Lemma 3 we obtain

$$
F_{0}(t)=i \sum_{j=1}^{m} \operatorname{Res}_{\rho=\rho_{j}}(1-s(\rho)) e^{i \rho t}=-i \sum_{j=1}^{m} \frac{\delta\left(-\rho_{j}\right)}{\dot{\delta}\left(\rho_{j}\right)} e^{i \rho_{j} t}=-\sum_{j=1}^{m} \alpha_{j} e^{i \rho_{j} t}=-F_{1}(t) .
$$

Thus,

$$
F(t) \equiv 0, \quad t>2 T
$$

Taking (35) into account we rewrite (34) as follows

$$
\theta(t)+F(t)+\int_{t}^{2 T} \theta(s-t) F(s) d s=0, \quad t \in(0,2 T) .
$$

In particular it yields $F(t) \in A C[0,2 T], F^{\prime}(t) \in L_{2}(0,2 T), F(2 T)=0$.

We note that the function $F(t)$ can be constructed from Eq. (36) which is usually better than to calculate $F(t)$ directly by $(32),(33),(29)$.

Acting in the same way as in [9] one can obtain that

$$
G(x, t)+F(x, t)+\int_{x}^{2 T-t} F(t+s) G(x, s) d s=0,0 \leq x \leq T, x<t<2 T-x .
$$

Equation (37) is called the Gel'fand-Levitan-Marchenko equation.

Now let us formulate physical realization conditions for the characteristic function $\triangle(\rho)$.

Theorem 3. For a function $\triangle(\rho)$ of the form (12) to be the characteristic function for a certain $R(x) \in B_{0}^{-}$, it is necessary ans sufficient that all zeros of $\triangle(\rho)$ in $\bar{\Pi}_{+}$are simple, have the form $\rho_{j}=i \tau_{j}, \tau_{j}>0, j=\overline{1, m}, m \geq 0$, and

$$
\alpha_{j}:=\frac{i \delta\left(-\rho_{j}\right)}{\dot{\delta}\left(\rho_{j}\right)}>0
$$

$R(x) \in B_{0}$ if and only if $\triangle(\rho)$ has no zeros in $\bar{\Pi}_{+}$, i.e. $m=0$. The specification of the characteristic function $\triangle(\rho)$ uniquely determines $R(x)$.

Proof. The necessity part of Theorem 1 was proved above. We prove the sufficiency. Put $\delta(\rho)=e^{i \rho T} \triangle(\rho)$. Then (25) holds, where $\theta(t)=\eta(T-t)$. Since for each fixed $x \geq 0$, the homogenious integral equation

$$
w(t)+\int_{x}^{\infty} F(t+s) w(s) d s=0, \quad t>x
$$

has only the trivial solution (see [9]), then Eq. (37) has a unique solution $G(x, t)$. The function $G(x, t)$ is absolutely continuous, $G(x, 2 T-x)=0$ and $\frac{d}{d x} G(x, x) \in L_{2}(0, T)$. We construct the function $e(x, \rho)$ by $(24)$.

Let us show that $e(0, \rho)=\delta(\rho)$. Indeed, it follows from (24) and (25) that

$$
e(0, \rho)-\delta(\rho)=\int_{0}^{2 T}(G(0, t)-\theta(t)) e^{i \rho t} d t .
$$

By virtue of (36) and (37),

$$
(G(0, t)-\theta(t))+\int_{0}^{2 T-t}(G(0, s)-\theta(s)) F(t+s) d s=0 .
$$


In view of (35), it gives us that the function $w(t):=G(0, t)-\theta(t)$ satisfies $(38)$ for $x=0$. Hence $G(0, t)=\theta(t)$, and according to $(39), e(0, \rho)=\delta(\rho)$.

Put $p(x):=-2 \frac{d G(x, x)}{d x}, x \in[0, T]$, and $p(x) \equiv 0, x>T$. It is easily shown that $-e^{\prime \prime}(x, \rho)+p(x) e(x, \rho)=\rho^{2} e(x, \rho), x>0$. We construct $R(x)$ by the formula

$$
R(x)=\frac{1}{u^{2}(x, 0)},
$$

where $u(x, \rho)=e^{-i \rho T} e(T-x, \rho)$. Since $\delta(0) \neq 0$, we have $u(T, 0) \neq 0$. It follows from (40) that $R(x) \in B_{0}^{-}$, where $0<x_{1}<\ldots<x_{p}<T$ are zeros of $u(x, 0)$. In particular, if $\delta(\rho)$ has no zeros in $\bar{\Pi}_{+}$, then, by virtue of Theorem $1, R(x) \in B_{0}$. The uniqueness of recovering $R(x)$ from $\triangle(\rho)$ was proved for example in [18].

Theorem 3 gives us the following algorithm for constructing $R(x)$ from the characteristic function $\triangle(\rho)$ :

Algorithm 1. Let a function $\triangle(\rho)$ satisfying the hypothesis of Theorem 3 be given. Then (1) Construct $F(t), t \in(0,2 T)$ from the integral equation (36), where $\theta(t)=\eta(T-t)$, or directly from (26), (29), (32), (33).

(2) Find $G(x, t)$ from the integral equation $(37)$.

(3) Calculate $R(x)$ by

$$
R(x)=\frac{1}{e^{2}(T-x)}, \quad e(x)=1+\int_{x}^{2 T-x} G(x, t) d t .
$$

Next we provide two other algortihms for the synthesis of the wave resistance from the characteristic function, which sometimes may give advantage from the numerical point of view.

Let $S(x, \lambda)$ be the solution of $(23)$ under the conditions $S(0, \lambda)=0, S^{\prime}(0, \lambda)=1$. Then

$$
S(x, \lambda)=\frac{\sin \rho x}{\rho}+\int_{0}^{x} Q(x, t) \frac{\sin \rho t}{\rho} d t
$$

where $Q(x, t)$ is a real, absolutely continuous function, $Q(x, 0)=0, Q(x, x)=\frac{1}{2} \int_{0}^{x} p(t) d t$.

Since $\langle e(x, \rho), S(x, \lambda)\rangle \equiv \delta(\rho)$ and $\delta\left(\rho_{j}\right)=0$, we get

$$
\epsilon\left(x, \rho_{j}\right)=e^{\prime}\left(0, \rho_{j}\right) S\left(x, \lambda_{j}\right), \quad \lambda_{j}=\rho_{j}^{2},
$$

and by virtue of (27) and (28),

$$
\beta_{j}:=\left(\int_{0}^{\infty} S^{2}\left(x, \lambda_{j}\right) d x\right)^{-1}=-\frac{2 \rho_{j} e^{\prime}\left(0, \rho_{j}\right)}{\dot{\delta}\left(\rho_{j}\right)}=-\frac{4 i \rho_{j}^{2}}{\delta\left(-\rho_{j}\right) \dot{\delta}\left(\rho_{j}\right)}>0 .
$$

Taking into account the relations

$$
\dot{\delta}\left(\rho_{j}\right)=e^{i \rho_{j} T} \dot{\triangle}\left(\rho_{j}\right), \delta\left(-\rho_{j}\right)=e^{-i \rho_{j} T} \triangle\left(-\rho_{j}\right)
$$


we obtain from $(44)$

$$
\beta_{j}=-\frac{4 i \rho_{j}^{2}}{\triangle\left(-\rho_{j}\right) \dot{\triangle}\left(\rho_{j}\right)}
$$

Consider the function

$$
a(x)=\sum_{j=1}^{m} \beta_{j} \frac{\cos \rho_{j} x}{\rho_{j}^{2}}+\frac{2}{\pi} \int_{0}^{\infty} \cos \rho x\left(\frac{1}{|\triangle(\rho)|^{2}}-1\right) d \rho .
$$

By virtue of $(12), \rho\left(\frac{1}{|\triangle(\rho)|^{2}}-1\right) \in L_{2}(0, \infty)$, and consequently $a(x)$ is absolutely continuous and $a^{\prime}(x) \in L_{2}$. The kernel $Q(x, t)$ from (42) satisfies the equation (see [10])

$$
f(x, t)+Q(x, t)+\int_{0}^{x} Q(x, s) f(s, t) d s=0, \quad x \geq 0, \quad 0<t<x,
$$

where $f(x, t)=\frac{1}{2}(a(x-t)-a(x+t))$. The function $R(x)$ can be constructed by the following algorithm:

Algorithm 2. Let $\triangle(\rho)$ be given. Then

(1) Construct $a(x)$ by (45).

(2) Find $Q(x, t)$ from the integral equation (46).

(3) Calculate $p(x):=2 \frac{d}{d x} Q(x, x)$.

(4) Construct

$$
R(x):=\frac{1}{e^{2}(T-x)},
$$

where $e(x)$ is the solution of the Cauchy problem $y^{\prime \prime}=p(x) y, y(T)=1, y^{\prime}(T)=0$.

Remark 2. We also can construct $R(x)$ using (6) and (8), namely:

$$
R(x)=\exp \left(2 \int_{0}^{x} h(t) d t\right),
$$

where $h(x)$ is the solution of the equation

$$
h(x)=\int_{0}^{x} h^{2}(t) d t+2 Q(T-x, T-x)-2 Q(T, T) .
$$

Remark 3. If $R(x) \in B_{0}$, then

$$
a(x)=\frac{2}{\pi} \int_{0}^{\infty} \cos \rho x\left(\frac{1}{|\triangle(\rho)|^{2}}-1\right) d \rho,
$$

and for constructing the solution of the inverse problem it is sufficient to specify $|\triangle(k)|$ for $k \geq 0$.

Now we provide an algorithm for the solution of the inverse problem which uses discrete spectral characteristics.

Let $X_{k}(x, \lambda), k=1,2$ be solutions of (7) under the conditions $X_{1}(0, \lambda)=X_{2}^{\prime}(0, \lambda)=$ $1, X_{1}^{\prime}(0, \lambda)=X_{2}(0, \lambda)=0$. Denote $\triangle_{k}(\lambda)=X_{k}(T, \lambda)$. Clearly,

$$
u(x, \rho)=X_{1}(x, \lambda)-i \rho X_{2}(x, \lambda), \triangle(\rho)=\triangle_{1}(\lambda)-i \rho \triangle_{2}(\Lambda),
$$


and consequently

$$
\triangle_{1}(\lambda)=\frac{\triangle(\rho)+\triangle(-\rho)}{2}, \triangle_{2}(\lambda)=\frac{\triangle(\rho)-\triangle(-\rho)}{2 i \rho} .
$$

Let $\left\{\mu_{n}\right\}_{n \geq 1}$ be zeros of $\triangle_{2}(\lambda)$, and

$$
\gamma_{n}:=\int_{0}^{T} X_{2}^{2}\left(x, \mu_{n}\right) d x>0
$$

It is easily shown that

$$
\gamma_{n}=\left(\triangle_{1}\left(\mu_{n}\right)\right)^{-1}\left(\frac{d}{d \lambda} \triangle_{2}(\lambda)\right)_{\mid \lambda=\mu_{n}}
$$

and

$$
\sqrt{\mu_{n}}=\frac{\pi n}{T}+\frac{1}{2 \pi n} \int_{0}^{T} q(\xi) d \xi+\frac{\omega_{n}}{n}, \quad \gamma_{n}=\frac{T^{3}}{2 n^{2} \pi^{2}}+\frac{\omega_{n 1}}{n^{3}}, \quad\left\{\omega_{n}\right\},\left\{\omega_{n 1}\right\} \in \ell_{2} .
$$

We introduce the function

$$
A(x)=\frac{2}{T} \sum_{n=1}^{\infty}\left(\frac{T \cos \sqrt{\mu_{n}} x}{2 \gamma_{n} \mu_{n}}-\cos \frac{\pi n x}{T}\right) .
$$

By virtue of (47), $A(x)$ is absolutely continuous, and $A^{\prime}(x) \in L_{2}$, It is known (see [9]) that

$$
X_{2}(x, \lambda)=\frac{\sin \rho x}{\rho}+\int_{0}^{x} D(x, t) \frac{\sin \rho t}{\rho} d t,
$$

where $D(x, t)$ is a real, absolutely continuous function, and $D(x, 0)=0, D(x, x)=$ $\frac{1}{2} \int_{0}^{x} q(t) d t$. The function $D(x, t)$ satisfies the equation (see [10])

$$
D(x, t)+B(x, t)+\int_{0}^{x} D(x, s) B(s, t) d s=0, \quad 0 \leq x \leq T, \quad 0<t<x,
$$

where $B(x, t)=\frac{1}{2}(A(x-t)-A(x+t))$. The function $R(x)$ can be constructed from the discrete data $\left\{\mu_{n}, \gamma_{n}\right\}_{n \geq 1}$ by the the following algorithm.

Algorithm 3. Let $\left\{\mu_{n}, \gamma_{n}\right\}_{n \geq 1}$ be given. Then

(1) Construct $A(x)$ by (48).

(2) Find $D(x, t)$ from the integral equation (49).

(3) Calculate $q(x):=2 \frac{d D(x, x)}{d x}$.

(4) Construct $R(x):=\frac{1}{u^{2}(x)}$, where $u(x)$ is the solution of the Cauchy problem $u^{\prime \prime}=$ $q(x) u, u(0)=1, u^{\prime}(0)=0$, or by

$$
R(x)=\exp \left(2 \int_{0}^{x} h(t) d t\right), h(x)=\int_{0}^{x} h^{2}(t) d t-2 D(x, x) .
$$

\section{Reconstruction of the transmission coefficients from their moduli.}


Lemma 4. Suppose that a function $\gamma(\rho)$ is regular in $\bar{\Pi}_{+}$, has no zeros in $\Pi_{+}$, and for $|\rho| \rightarrow \infty, \rho \in \bar{\Pi}_{+}, \gamma(\rho)=1+O\left(\frac{1}{\rho}\right)$. Let $\gamma(k)=|\gamma(k)| e^{-i \beta(k)}, k=\operatorname{Re} \rho$. Then

$$
\beta(k)=\frac{1}{\pi} \int_{-\infty}^{\infty} \frac{\ln |\gamma(\xi)|}{\xi-k} d \xi
$$

In (50) (and everywhere below, where necessary) the integral is understood in the principal value sense.

Proof. First we suppose that $\gamma(k) \neq 0$ for real $k$. By Cauchy's theorem, taking into account the hypothesis of the lemma, we obtain

$$
\frac{1}{2 \pi i} \int_{C_{r, \epsilon}} \frac{\ln \gamma(\xi)}{\xi-k} d \xi=0
$$

where $C_{r, \epsilon}$ is the closed contour (with counterclockwise circuit) consisting of the semicircles $C_{r}=\left\{\xi: \xi=r e^{i \varphi}, \quad \varphi \in[0, \pi]\right\}, \quad \Gamma_{\epsilon}=\left\{\xi: \xi-k=\epsilon e^{i \varphi}, \quad \varphi \in[0, \pi]\right\}$ and the intervals $\xi \in[-r, r] \backslash[k-\epsilon, k+\epsilon]$ of the real axis. Since

$$
\begin{gathered}
\lim _{\epsilon \rightarrow 0} \frac{1}{2 \pi i} \int_{\Gamma_{\epsilon}} \frac{\ln \gamma(\xi)}{\xi-k} d \xi=-\frac{1}{2} \ln \gamma(k), \\
\lim _{r \rightarrow \infty} \frac{1}{2 \pi i} \int_{C_{r}} \frac{\ln \gamma(\xi)}{\xi-k} d \xi=0,
\end{gathered}
$$

we get from (51) that

$$
\ln \gamma(k)=\frac{1}{\pi i} \int_{-\infty}^{\infty} \frac{\ln \gamma(\xi)}{\xi-k} d \xi
$$

Separating here real and imaginary parts, we arrive at (50).

Suppose now that for real $\rho$, the function $\gamma(\rho)$ has one zero $\rho_{0}=0$ of multiplicity $s$ (the general case is treated in the same way). Denote

$$
\tilde{\gamma}(\rho)=\gamma(\rho)\left(\frac{\rho+i \epsilon}{\rho}\right)^{s}, \epsilon>0 ; \tilde{\gamma}(k)=|\tilde{\gamma}(k)| e^{-i \tilde{\beta}(k)} .
$$

Then

$$
\beta(k)=\tilde{\beta}(k)+\operatorname{sarctg} \frac{\epsilon}{k} .
$$

For the function $\tilde{\gamma}(\rho),(50)$ has been proved. Hence, (52) takes the form

$$
\beta(k)=\frac{1}{\pi} \int_{-\infty}^{\infty} \frac{\ln |\gamma(\xi)|}{\xi-k} d \xi+\frac{s}{2 \pi} \int_{-\infty}^{\infty} \frac{\ln \left(1+\frac{\epsilon^{2}}{\xi^{2}}\right)}{\xi-k} d \xi+\operatorname{sarctg} \frac{\epsilon}{k} .
$$

When $\epsilon \rightarrow 0$, it gives us $(50)$. Lemma 4 is proved.

Lemma 5. Suppose that a function $\gamma(\rho)$ is regular in $\bar{\Pi}_{+}, \gamma(-\bar{\rho})=\overline{\gamma(\rho)}$, and for $|\rho| \rightarrow$ $\infty, \rho \in \bar{\Pi}_{+}, \gamma(\rho)=1+O\left(\frac{1}{\rho}\right)$. Let $\gamma(k)=|\gamma(k)| e^{-i \beta(k)}$, and let $\rho_{j}=k_{j}+i \tau_{j}, \tau_{j}>0, j=$ $\overline{1, s}$ be zeros of $\gamma(\rho)$ in $\Pi_{+}$. Then

$$
\beta(k)=\frac{1}{\pi} \int_{-\infty}^{\infty} \frac{\ln |\gamma(\xi)|}{\xi-k} d \xi+2 \sum_{j=1}^{s} \operatorname{arctg} \frac{\tau_{j}}{k-k_{j}} .
$$


Proof. Since $\gamma(-\bar{\rho})=\overline{\gamma(\rho)}$, zeros of $\gamma(\rho)$ in $\Pi_{+}$are symmetrical with respect to the imaginary axis. If $\rho_{j}=i \tau_{j}, \tau_{j}>0$, then

$$
\arg \frac{k+\rho_{j}}{k-\rho_{j}}=2 \operatorname{arctg} \frac{\tau_{j}}{k}
$$

If $\rho_{j}=k_{j}+i \tau_{j}, k_{j}>0, \tau_{j}>0$, then

$$
\arg \frac{\left(k+\rho_{j}\right)\left(k-\bar{\rho}_{j}\right)}{\left(k-\rho_{j}\right)\left(k+\bar{\rho}_{j}\right)}=2 \operatorname{arctg} \frac{\tau_{j}}{k-k_{j}}+2 \operatorname{arctg} \frac{\tau_{j}}{k+k_{j}} .
$$

Denote

$$
\tilde{\gamma}(\rho)=\gamma(\rho) \prod_{j=1}^{s} \frac{\rho+\rho_{j}}{\rho-\rho_{j}} .
$$

The function $\tilde{\gamma}(\rho)$ satisfies the hypothesis of Lemma 4. Then using (50), (54) and (55) we arrive at $(53)$.

Using Lemma 5 one can construct the transmission coefficients from their moduli and information about their zeros in $\Pi_{+}$. For definiteness we confine ourselves to the case $h \neq 0$.

Theorem 4. Let

$$
f_{1}(k)=\alpha_{1}(k) e^{-i \delta_{1}(k)},
$$

and let $\rho_{j}^{*}=i \tau_{j}^{*}, \tau_{j}^{*}>0, j=\overline{1, m}^{*}$ be zeros of $f_{1}(\rho)$ in $\Pi_{+}$. Then

$$
\delta_{1}(k)=k T+\frac{1}{\pi} \int_{-\infty}^{\infty} \frac{\ln \alpha_{1}(\xi)}{\xi-k} d \xi+2 \sum_{j=1}^{m^{*}} \operatorname{arctg} \frac{\tau_{j}^{*}}{k} .
$$

In particular, if $R(x) \in B_{0}$, then

$$
\delta_{1}(k)=k T+\frac{1}{\pi} \int_{-\infty}^{\infty} \frac{\ln \alpha_{1}(\xi)}{\xi-k} d \xi
$$

Proof. Denote $\gamma(\rho)=e^{i \rho T} f_{1}(\rho)$. If follows from (15) that

$$
\gamma(\rho)=1+\int_{-T}^{T} g_{1}(t) e^{i \rho(T-t)} d t
$$

and consequently, $\gamma(-\bar{\rho})=\overline{\gamma(\rho)}$, and for $|\rho| \rightarrow \infty, \rho \in \bar{\Pi}_{+}, \gamma(\rho)=1+O\left(\frac{1}{\rho}\right)$. Thus, the function $\gamma(\rho)$ satisfies the hypothesis of Lemma 5. Using (53) and the relations $|\gamma(k)|=$ $\alpha_{1}(k), \delta_{1}(k)=\beta(k)+k T$, we arrive at $(57)$.

Similarly we prove the following theorem.

Theorem 5. Let

$$
f_{2}(k)=\alpha_{2}(k) e^{-i \delta_{2}(k)},
$$

and let $\rho_{j}^{0}=k_{j}^{0}+i \tau_{j}^{0}, \quad \tau_{j}^{0}>0, j=\overrightarrow{1, m}^{0}$ be zeros of $f_{2}(\rho)$ in $\Pi_{+}$. Then

$$
\delta_{2}(k)=\frac{\pi}{2} \operatorname{sign}\left(\frac{k}{h}\right)+k T+\frac{1}{\pi} \int_{-\infty}^{\infty} \frac{\ln \left|\frac{2 \xi}{h} \alpha_{2}(\xi)\right|}{\xi-k} d \xi+2 \sum_{j=1}^{m_{0}} \operatorname{arctg} \frac{\tau_{j}^{0}}{k-k_{j}^{0}} .
$$


In particular, if $f_{2}(\rho)$ has no zeros in $\Pi_{+}$, then

$$
\delta_{2}(k)=\frac{\pi}{2} \operatorname{sign}\left(\frac{k}{h}\right)+k T+\frac{1}{\pi} \int_{-\infty}^{\infty} \frac{\ln \left|\frac{2 \xi}{h} \alpha_{2}(\xi)\right|}{\xi-k} d \xi .
$$

Thus, the specification of $\alpha_{j}(k)$ uniquely determines the transmission coefficients only when they have no zeros in $\Pi_{+}$. In particular, for the class $B_{0}, f_{1}(\rho)$ is uniquely determined by its modulus, and all possible transmission coefficients $f_{2}(\rho)$ can be constructed by means of solving the integral equation (21).

\section{Symmetrical case.}

The wave resistance is called symmetrical if $R(T-x)=R(x)$. For the symmetrical case the transmission coefficient $f_{2}(k)$ is uniquely determined (up to the sign) from its modulus.

Theorem 6. For the wave resistance to be symmetrical it is necessary and sufficient that $\operatorname{Re} f_{2}(k)=0$. At that, $f_{2}(k)=-f_{2}(-k), g_{2}(t)=-g_{2}(-t)$, and

$$
f_{2}(k)=-2 i \int_{0}^{T} g_{2}(t) \sin k t d t
$$

Proof. According to (9) und (5),

$$
f_{2}(\rho)=\frac{1}{2 i \rho}\left(u^{\prime}(T, \rho)+i \rho u(T, \rho)+h u(T, \rho)\right) .
$$

Since $u(x, \rho)=X_{1}(x, \lambda)-i \rho X_{2}(x, \lambda)$, we calculate

$$
\operatorname{Re} f_{2}(k)=\frac{1}{2}\left(X_{1}(T, \lambda)-X_{2}^{\prime}(T, \lambda)-h X_{2}(T, \lambda)\right)
$$

If $R(x)=R(T-x)$, then it follows from (6) and (8) that $h(x)=-h(T-x), q(x)=q(T-x)$, and consequently $h=0, X_{1}(T, \lambda) \equiv X_{2}^{\prime}(T, \lambda)$ (see [19]), i.e. $\operatorname{Re} f_{2}(k)=0$.

Conversly, if $\operatorname{Re} f_{2}(k)=0$, then $(62)$ gives us

$$
X_{1}(T, \lambda)-X_{2}^{\prime}(T, \lambda)-h X_{2}(T, \lambda) \equiv 0 .
$$

Since for $k \rightarrow \infty$,

$$
X_{2}\left(T, k^{2}\right)=\frac{\sin k T}{k}+O\left(\frac{1}{k^{2}}\right), X_{1}(T, \lambda)-X_{2}^{\prime}(T, \lambda)=O\left(\frac{1}{k^{2}}\right),
$$

we obtain from $(63)$ that $h=0, X_{1}(T, \lambda) \equiv X_{2}^{\prime}(T, \lambda)$. Consequently, $q(x)=q(T-x)$ (see [19]). Similarly one can prove that $g(x)=g(T-x)$. Hence $h(x)=-h(T-x)$, and $R(x)=R(T-x)$.

Further, it follows from (16) that

$$
\operatorname{Re} f_{2}(k)=\int_{-T}^{T} g_{2}(t) \cos k t d t, \quad \operatorname{Im} f_{2}(k)=-\int_{-T}^{T} g_{2}(t) \sin k t d t .
$$

For the symmetrical case $\operatorname{Re} f_{2}(k)=0$, and consequently $g_{2}(t)=-g_{2}(-t)$. Then

$$
f_{2}(k)=i \operatorname{Im} f_{2}(k)=-2 i \int_{0}^{T} g_{2}(t) \sin k t d t, \quad f_{2}(-k)=-f_{2}(k) .
$$


Theorem 6 is proved.

Thus, $f_{2}(k)$ can be constructed (up to the sign) by formulas $\operatorname{Re} f_{2}(k)=0, \quad\left|\operatorname{Im} f_{2}(k)\right|=$ $\alpha_{2}(k), \quad f_{2}(k)=-f_{2}(-k)$.

\section{Synthesis of the wave resistance from the power reflection coefficient.}

In this section, using results obtained above, we provide a procedure for constructing $R(x)$ from the given $\sigma(k)$. For definiteness we confine ourselves to the case $h \neq 0$. Let the power reflection coefficient $\sigma(k)(0 \leq \sigma(k)<1, \sigma(k)=\sigma(-k))$ be given. Our scheme of calculation is:

Step 1. Calculate $\alpha_{1}(k)$ and $\alpha_{2}(k)$ by the formula

$$
\alpha_{1}^{2}(k)=1+\alpha_{2}^{2}(k)=\frac{1}{1-\sigma(k)} .
$$

Step 2. Construct $f_{1}(k)$ by $(56),(57)$ or for $R(x) \in B_{0}$ by $(56)$, (58). Find $g_{1}(t)$ from the relation

$$
\int_{-T}^{T} g_{1}(t) e^{-i k t} d t=f_{1}(k)-e^{i k T} .
$$

Step 3. Construct $f_{2}(k)$ by $(59),(60)$ or, if $f_{2}(\rho)$ has no zeros in $\Pi_{+}$, by $(59),(61)$. Find $g_{2}(t)$ from the relation

$$
\int_{-T}^{T} g_{2}(t) e^{-i k t} d t=f_{2}(k)
$$

We note that $g_{2}(t)$ can be constructed also from the integral equation (21). In this case $f_{2}(k)$ is calculated by $(16)$.

Step 4. Calculate $\eta(t)=g_{1}(t)+g_{2}(t)$ and $\triangle(\rho)$ by $(12)$.

Step 5. Construct $R(x)$ using one of the aboveformulated Algorithms $1-3$.

Remark 4. In some concrete algorithms it is not necessary to make all calculations from above. For example, let $R(x) \in B_{0}$, and we use Algorithm 2. Then it is not necessary to calculate $g_{1}(t), g_{2}(t)$ and $\eta(t)$, since we need $|\triangle(k)|$ only.

Now we consider a concrete algorithm which realizes this scheme. For simplicity, we consider the case $R(x) \in B_{0}$.

For $t \in[0, T]$ we consider the functions

$$
\begin{gathered}
\varphi_{j}(t)=g_{j}(t)+g_{j}(-t), \quad \psi_{j}(t)=g_{j}(t)-g_{j}(-t), j=1,2, \\
\varphi(t)=\eta(t)+\eta(-t), \quad \psi(t)=\eta(t)-\eta(-t) .
\end{gathered}
$$

Since $\eta(t)=g_{1}(t)+g_{2}(t)$, we get

$$
\psi(t)=\varphi_{1}(t)+\varphi_{2}(t), \quad \psi(t)=\psi_{1}(t)+\psi_{2}(t) .
$$

Solving $(65)$ and $(66)$ with respect to $g_{j}(t)$ and $\eta(t)$ we obtain

$$
g_{j}(t)=\left\{\begin{array}{ll}
\frac{1}{2}\left(\varphi_{j}(t)+\psi_{j}(t)\right) & , t>0, \\
\frac{1}{2}\left(\varphi_{j}(-t)-\psi_{j}(-t)\right) & , \quad t<0,
\end{array} \quad \eta(t)= \begin{cases}\frac{1}{2}(\varphi(t)+\psi(t)), & t>0 \\
\frac{1}{2}(\varphi(-t)+\psi(-t)), & t<0\end{cases}\right.
$$


It follows from (17) and (65) that

$$
\varphi_{1}(T)=\psi_{1}(T)=-w_{1}, \quad \varphi_{2}(T)=\psi_{2}(T)=-\frac{h}{2}, \quad \psi_{1}(0)=\psi_{2}(0)=0,
$$

where

$$
w_{1}=-\frac{1}{2} \int_{0}^{T} h^{2}(\xi) d \xi
$$

By virtue of Lemma 3 ,

$$
\left.\begin{array}{l}
f_{1}(k)=\left(\cos k T+C_{1}(k)\right)-i\left(\sin k T+S_{1}(k)\right), \\
f_{2}(k)=C_{2}(k)-i S_{2}(k), \\
\triangle(k)=((\cos k T+C(k))-i(\sin k T+S(k)),
\end{array}\right\}
$$

where

$$
\begin{aligned}
C_{j}(k) & =\int_{0}^{T} \varphi_{j}(t) \cos k t d t, \quad S_{j}(k)=\int_{0}^{T} \psi_{j}(t) \sin k t d t \\
C(k) & =\int_{0}^{T} \varphi(t) \cos k t d t, \quad S(k)=\int_{0}^{T} \psi(t) \sin k t d t
\end{aligned}
$$

Using (69) and (71) we obtain the following asymptotic formulas for $C_{j}(k)$ and $S_{j}(k)$ as $k \rightarrow \infty$

$$
\left.\begin{array}{ll}
C_{1}(k)=-w_{1} \frac{\sin k T}{k}+\frac{\omega(k)}{k}, & C_{2}(k)=-\frac{h}{2} \frac{\sin k T}{k}+\frac{\omega(k)}{k}, \\
S_{1}(k)=w_{1} \frac{\cos k T}{k}+\frac{\omega(k)}{k}, & S_{2}(k)=\frac{h}{2} \frac{\cos k T}{k}+\frac{\omega(k)}{k} .
\end{array}\right\}
$$

Here and below, one and the same symbol $\omega(k)$ denotes various functions from $L_{2}(-\infty, \infty)$. Comparing (70) with the relations $f_{j}(k)=\alpha_{j}(k) e^{-i \delta_{j}(k)}$, we derive

$$
\begin{gathered}
C_{1}(k)=\alpha_{1}(k) \cos \delta_{1}(k)-\cos k T, \quad S_{1}(k)=\alpha_{1}(k) \sin \delta_{1}(k)-\sin k T, \\
C_{2}(k)=\alpha_{2}(k) \cos \delta_{2}(k), \quad S_{2}(k)=\alpha_{2}(k) \sin \delta_{2}(k) .
\end{gathered}
$$

For calculating the arguments of the transmission coefficients we will use (58) and (61), i.e.

$$
\delta_{1}(k)=k T+\widetilde{\delta}_{1}(k), \quad \delta_{2}(k)=\frac{\pi}{2} w+k T+\widetilde{\delta}_{2}(k), \quad k>0,
$$

where $w=\operatorname{sign} h$,

$$
\begin{gathered}
\tilde{\delta}_{1}(k)=\frac{1}{\pi} \int_{-\infty}^{\infty} \frac{\ln \alpha_{1}(\xi)}{\xi-k} d \xi \\
\tilde{\delta}_{2}(k)=\frac{1}{\pi} \int_{-\infty}^{\infty} \frac{\ln \tilde{\alpha}_{2}(\xi)}{\xi-k} d \xi, \quad \tilde{\alpha}_{2}(\xi):=\left|\frac{2 \xi}{h} \alpha_{2}(\xi)\right| .
\end{gathered}
$$

It follows from (73) - (75) and (20) that for $k \rightarrow+\infty$

$$
\delta_{1}(k)=k T+\frac{w_{1}}{k}+\frac{\omega(k)}{k}, \delta_{2}(k)=\frac{\pi}{2} w+k T+\omega(k) .
$$

Substituting (76) into (74) and (75), we calculate for $k>0$

$$
\left.\begin{array}{l}
C_{1}(k)=\cos k T\left(\alpha_{1}(k) \cos \tilde{\delta}_{1}(k)-1\right)-\sin k T\left(\alpha_{1}(k) \sin \tilde{\delta}_{1}(k)\right), \\
S_{1}(k)=\sin k T\left(\alpha_{1}(k) \cos \tilde{\delta}_{1}(k)-1\right)+\cos k T\left(\alpha_{1}(k) \sin \tilde{\delta}_{1}(k)\right),
\end{array}\right\}
$$




$$
\left.\begin{array}{l}
C_{2}(k)=-\alpha_{2}(k) w\left(\sin k T \cos \tilde{\delta}_{2}(k)+\cos k T \sin \tilde{\delta}_{2}(k)\right), \\
S_{2}(k)=\alpha_{2}(k) w\left(\cos k T \cos \tilde{\delta}_{2}(k)-\sin k T \sin \tilde{\delta}_{2}(k)\right) .
\end{array}\right\}
$$

Furhter, consider the functions

$$
\left.\begin{array}{l}
\varphi_{1}^{*}(t)=\varphi_{1}(t)+w_{1}, \quad \psi_{1}^{*}(t)=\psi_{1}(t)+w_{1} \frac{t}{T}, \\
\varphi_{2}^{*}(t)=\varphi_{2}(t)+\frac{h}{2}, \quad \psi_{2}^{*}(t)=\psi_{2}(t)+\frac{h}{2} \cdot \frac{t}{T} .
\end{array}\right\}
$$

Then in view of (69),

$$
\varphi_{j}^{*}(T)=\psi_{j}^{*}(T)=\psi_{j}^{*}(0)=0, \quad j=1,2
$$

Denote

$$
C_{j}^{*}(k)=\int_{0}^{T} \varphi_{j}^{*}(t) \cos k t d t, \quad S_{j}^{*}(k)=\int_{0}^{T} \psi_{j}^{*}(t) \sin k t d t .
$$

Integrating by parts the integrals in (84) and taking (83) into account, we obtain

$$
C_{j}^{*}(k)=-\int_{0}^{T} \varphi_{j}^{*^{\prime}}(t) \frac{\sin k T}{k} d t, \quad S_{j}^{*}(k)=\int_{0}^{T} \psi_{j}^{*^{\prime}}(t) \frac{\cos k t}{k} d t
$$

Clearly,

$$
\begin{gathered}
C_{1}(k)=C_{1}^{*}(k)-w_{1} \frac{\sin k T}{k}, S_{1}(k)=S_{1}^{*}(k)+w_{1}\left(\frac{\cos k T}{k}-\frac{\sin k T}{T k^{2}}\right), \\
C_{2}(k)=C_{2}^{*}(k)-\frac{h}{2} \frac{\sin k T}{k}, S_{2}(k)=S_{2}^{*}(k)+\frac{h}{2}\left(\frac{\cos k T}{k}-\frac{\sin k T}{T k^{2}}\right) .
\end{gathered}
$$

Now let the power reflection coefficient $\sigma(k)(0 \leq \sigma(k)<1, \sigma(k)=\sigma(-k))$ be given for $|k| \leq B$, and put

$$
\sigma(k)=\frac{h^{2}}{4 k^{2}+h^{2}},|k|>B,
$$

where $B>|h|$ is chosen sufficiently large, such that (see $(20)) \sigma(k)$ is sufficiently accurate for $|k|>B$.

Using (64) we calculate $\alpha_{1}(k)$ and $\alpha_{2}(k)$. Then $\alpha_{1}^{2}(k)-\alpha_{2}^{2}(k)=1, \alpha_{j}(k)=\alpha_{j}(-k)>0$, and

$$
\alpha_{1}^{2}(k)=1+\frac{h^{2}}{4 k^{2}}, \alpha_{2}^{2}(k)=\frac{h^{2}}{4 k^{2}},|k|>B .
$$

To calculate $\tilde{\delta}_{1}(k)$ we use $(77)$. First let $|k|>B+\chi, \chi>0$. In view of (89), the equality (77) takes the form

$$
\widetilde{\delta}_{1}(k)=\frac{1}{\pi} \int_{-B}^{B} \frac{\ln \alpha_{1}(\xi)}{\xi-k} d \xi+\frac{1}{2 \pi} \int_{|\xi|>B} \frac{\ln \left(1+\frac{h^{2}}{4 \xi^{2}}\right)}{\xi-k} d \xi .
$$

Since

$$
\begin{aligned}
& \frac{1}{2 \pi} \int_{|\xi|>B} \frac{\ln \left(1+\frac{h^{2}}{4 \xi^{2}}\right)}{\xi-k} d \xi=\frac{1}{2 \pi} \sum_{j=1}^{\infty} \frac{(-1)^{j-1}}{j}\left(\frac{h^{2}}{4}\right)^{j} \int_{|\xi|>B} \frac{d \xi}{\xi^{2 j}(\xi-k)}= \\
& =\frac{1}{2 \pi} \sum_{j=1}^{\infty} \frac{(-1)^{j-1}}{j}\left(\frac{h^{2}}{4}\right)^{j} \int_{|\xi|>B}\left\{\frac{1}{k^{2 j}}\left(\frac{1}{\xi-k}-\frac{1}{\xi}\right)-\sum_{s=1}^{2 j-1} \frac{1}{k^{2 j-s} \xi^{s+1}}\right\} d \xi,
\end{aligned}
$$


we get

$$
\begin{aligned}
& \tilde{\delta}_{1}(k)=\frac{1}{\pi} \int_{-B}^{B} \frac{\ln \alpha_{1}(\xi)}{\xi-k} d \xi+\frac{1}{2 \pi} \ln \left(1+\frac{2 B}{k-B}\right) \ln \left(1+\frac{h^{2}}{4 k^{2}}\right)+ \\
& +\frac{1}{\pi} \sum_{j=1}^{\infty} \frac{(-1)^{j}}{j}\left(\frac{h^{2}}{4}\right)^{j} \sum_{\mu=0}^{j-1} \frac{1}{(2 j-2 \mu-1) B^{2 j-2 \mu-1} k^{2 \mu+1}} .
\end{aligned}
$$

Using the relation

$$
\frac{1}{\pi} \int_{-B}^{B} \frac{\ln \alpha_{1}(\xi)}{\xi-k} d \xi=-\frac{1}{k \pi} \int_{-B}^{B} \ln \alpha_{1}(\xi) d \xi+\frac{1}{\pi} \int_{-B}^{B} \frac{\xi \ln \alpha_{1}(\xi)}{k(\xi-k)} d \xi
$$

we separate in $(90)$ the terms of order $1 / k$, i.e.

$$
\tilde{\delta}_{1}(k)=\frac{w_{1}}{k}+\tilde{\delta}_{1}^{*}(k),
$$

where

$$
\begin{gathered}
\tilde{\delta}_{1}^{*}(k)=\frac{1}{\pi} \int_{-B}^{B} \frac{\xi \ln \alpha_{1}(\xi)}{k(\xi-k)} d \xi+\frac{1}{2 \pi} \ln \left(1+\frac{2 B}{k-B}\right) \ln \left(1+\frac{h^{2}}{4 k^{2}}\right)+ \\
+\frac{1}{\pi} \sum_{j=1}^{\infty} \frac{(-1)^{j}}{j}\left(\frac{h^{2}}{4}\right)^{j} \sum_{\mu=1}^{j-1} \frac{1}{(2 j-2 \mu-1) B^{2 j-2 \mu-1} k^{2 \mu+1}},|k|>B+\chi \\
w_{1}=-\frac{1}{\pi} \int_{-B}^{B} \ln \alpha_{1}(\xi) d \xi+\frac{1}{\pi} \sum_{j=1}^{\infty} \frac{(-1)^{j}}{j}\left(\frac{h^{2}}{4 B^{2}}\right)^{j} \cdot \frac{B}{2 j-1}<0
\end{gathered}
$$

It is easy to show that

$$
\left|\tilde{\delta}_{1}^{*}(k)\right| \leq \frac{\Omega_{1}^{*}}{k^{2}}
$$

where

$$
\Omega_{1}^{*}=\frac{B+\chi}{\pi \chi} \int_{0}^{B} \xi \ln \alpha_{1}(\xi) d \xi+\frac{h^{2}}{8 \pi} \ln \left(1+\frac{2 B}{\chi}\right)+\frac{h^{2}}{2 \pi} .
$$

Now let $|k| \leq B+\chi$. In this case we rewrite (77) in the form

$$
\tilde{\delta}_{1}(k)=\frac{1}{\pi} \int_{0}^{\infty} \frac{\ln \alpha_{1}(\xi+k)-\ln \alpha_{1}(\xi-k)}{\xi} d \xi .
$$

Take $r \geq 2 B+\chi$. Since

$$
\left|\ln \left(1+\frac{h^{2}}{4 \xi^{2}}\right)-\frac{h^{2}}{4 \xi^{2}}\right| \leq \frac{h^{4}}{32 \xi^{4}},
$$

we get

$$
\frac{1}{\pi} \int_{r}^{\infty} \frac{\ln \alpha_{1}(\xi+k)-\ln \alpha_{1}(\xi-k)}{\xi} d \xi=\frac{h^{2}}{8 \pi} \int_{r}^{\infty} \frac{1}{\xi}\left(\frac{1}{(\xi+k)^{2}}-\frac{1}{(\xi-k)^{2}}\right) d \xi+\epsilon(k),
$$

where

$$
|\epsilon(k)| \leq \frac{h^{4}}{96 \pi r(r-B-\chi)^{3}}
$$


Calculating this integral and substituting into (94), we obtain

$$
\begin{aligned}
& \tilde{\delta}_{1}(k)=\frac{1}{\pi} \int_{0}^{r} \frac{\ln \alpha_{1}(\xi+k)-\ln \alpha_{1}(\xi-k)}{\xi} d \xi+\frac{h^{2}}{8 \pi}\left(\frac{1}{k^{2}} \ln \frac{r+k}{r-k}-\frac{1}{k}\left(\frac{1}{R+k}+\frac{1}{R-k}\right)\right)+ \\
& +\epsilon(k), \widetilde{\delta}_{1}(0)=0,|k| \leq B+\chi, r \geq 2 B+\chi .
\end{aligned}
$$

In order to calculate $\delta_{2}(k)$ we use (78). Let $|k|>B+\chi, \chi>0$. According to (89) we have $\tilde{\alpha}_{2}(k)=1$ for $|k|>B$. Hence

$$
\tilde{\delta}_{2}(k)=\frac{1}{\pi} \int_{-B}^{B} \frac{\ln \tilde{\alpha}_{2}(\xi)}{\xi-k} d \xi,|\xi|>B+\chi .
$$

For $|k| \leq B+\chi$ it is more convenient to use another formula:

$$
\tilde{\delta}_{2}(k)=\frac{1}{\pi} \int_{0}^{r} \frac{\ln \tilde{\alpha}_{2}(\xi+k)-\ln \tilde{\alpha}_{2}(\xi-k)}{\xi} d \xi,|k| \leq B+\chi, r=2 B+\chi .
$$

Let us calculate $\psi_{j}^{*}(t), \varphi_{j}^{*}(t)$. By virtue of $(84)$,

$$
\begin{aligned}
\psi_{j}^{*}(t) & =\frac{2}{T} \sum_{n=1}^{\infty} S_{j}^{*}\left(\frac{n \pi}{T}\right) \sin \frac{n \pi}{T} t, \\
\varphi_{j}^{*}(t) & =\frac{1}{T} C_{j}^{*}(0)+\frac{2}{T} \sum_{n=1}^{\infty} C_{j}^{*}\left(\frac{n \pi}{T}\right) \cos \frac{n \pi}{T} t .
\end{aligned}
$$

According to (85), the series in (98) converge absolutely and uniformly. By virtue of (80), (81), (86), (87), the coefficients $S_{j}^{*}\left(\frac{n \pi}{T}\right)$ and $C_{j}^{*}\left(\frac{n \pi}{T}\right)$ can be calculated via the formulas

$$
\left.\begin{array}{ll}
S_{1}^{*}(k)=(-1)^{n}\left(\alpha_{1}(k) \sin \tilde{\delta}_{1}(k)-\frac{w_{1}}{k}\right), & k=\frac{n \pi}{T}, n \geq 1, \\
S_{2}^{*}(k)=(-1)^{n}\left(\alpha_{2}(k) w \cos \tilde{\delta}_{2}(k)-\frac{h}{2 k}\right), & k=\frac{n \pi}{T}, n \geq 1, \\
C_{1}^{*}(k)=(-1)^{n}\left(\alpha_{1}(k) \cos \tilde{\delta}_{1}(k)-1\right)+\delta_{n 0} w_{1} T, & k=\frac{n \pi}{T}, n \geq 0, \\
C_{2}^{*}(k)=(-1)^{n+1} w \sin \tilde{\delta}_{2}(k)+\delta_{n 0} \frac{h T}{2}, & k=\frac{n \pi}{T}, n \geq 0,
\end{array}\right\}
$$

where $\delta_{n j}$ is the Kronecker delta.

Using the formulas obtained above we arrive at the following numerical algorithm for the solution of Inverse Problem 1.

Algorithm 4. Let the power reflection coefficient $\sigma(k)$ be given for $|k| \leq B$, and take $\sigma(k)$ according to (88) for $|k|>B$. Then

(1) Construct $\alpha_{1}(k)$ and $\alpha_{2}(k)$ by $(64)$.

(2) Find the number $w_{1}$ by $(93)$.

(3) Calculate $\tilde{\delta}_{1}(k), \widetilde{\delta}_{2}(k)$ by $(91),(92),(96)$ for $|k|>B+\chi$, and by $(95),(97)$ for $|k| \leq B+\chi$.

(4) Construct $\varphi_{j}^{*}(t), \psi_{j}^{*}(t)$ by $(98)$, where $S_{j}^{*}\left(\frac{n \pi}{T}\right), C_{j}^{*}\left(\frac{n \pi}{T}\right)$ is defined by $(99)$.

$(5)$ Find $\varphi_{j}(t), \psi_{j}(t)$ using $(82)$. 
(6) Calculate $\eta(t), t \in[-T, T]$ by $(67),(68)$.

(7) Find $F(t), 0<t<2 T$ from the integral equation (36), where $\theta(t)=\eta(T-t)$.

(8) Calculate $G(x, t)$ from the integral equation (37).

(9) Construct $R(x), x \in[0, T]$ via (41).

Remark 5. This algorithm is one of several possible numerical algorithms for the solution of Inverse Problem 1. Using the obtained results one can construct various algorithms for synthesizing $R(x)$ from spectral characteristics.

Acknowledgment. The authors gratefully acknowledge support from Volkswagen Foundation. 


\section{References}

1. Sveshnikov, A. G. and Il'inskii, A.S., Design problems in electrodynamics, Dokl. Akad. Nauk SSSR, 204 (1972), 5, 1077 - 1080.

2. Tikhonravov, A.V., The accuracy obtainable in principle when solving synthesis problems, Zh. Vychisl. Mat. mat. Fiz. 22 (1982), 6, 1421 - 1433; English transl. in USSR Comput. Maths. Math. Phys. 22 (1982), 6, $143-157$.

3. Meshanov, V.P. and Feldstein, A.L., Automatic Design of Directional Couplers, Moscow: Sviaz', 1980 (in Russian).

4. Litvinenko, O.N. and Soshnikov, V.I., The Theory of Heterogenious Lines and their Applications in Radio Engineering, Moscow: Radio, 1964 (in Russian).

5. Hurt, N.E., Phase Retrieval and Zero Crossing, Dordrecht: Kluwer, 1989.

6. Bates, R.H.T. and McDonnell, M.J., Image Restoration and Reconstruction, Oxford: Clarendon, 1986.

7. Hoenders, B.J., On the solution of the phase retrieval problem, J. Math. Phys. 16 (1975), $1719-1725$.

8. Borg, G., Eine Umkehrung der Sturm-Liouvilleschen Eigenwertaufgabe, Acta Math. 78 (1946), 1 - 96.

9. Marchenko, V.A., Sturm-Liouville Operators and their Applications, Kiev: Naukova Dumka, 1977, (Engl. transl. 1986 (Basel: Birkhäuser)).

10. Levitan, B.M., Inverse Sturm-Liouville Problems, Moscow: Nauka, 1984, (Engl. transl. 1987 (Utrecht: VNU Science Press)).

11. Pöschel, J. and Trubowitz, E., Inverse Spectral Theory, New York: Academic, 1987.

12. McLaughlin, Analytical methods for recovering coeffitients in differential equations from spectral data, STAM Rev. 28 (1986), 53 - 72.

13. Freiling, G. and Yurko, V., Inverse problems for differential equations with turning points, Inverse Problems 13 (1997), 1247 - 1263.

14. Wasow, W., Linear Turning Point Theory, Berlin: Springer, 1985.

15. McHugh, J.M., An historical survey of ordinary linear differential equations with a large parameter and turning points, Arch. Hist. Exact Sci 7 (1971), $277-324$.

16. Eberhard, W., Freiling, G. and Schneider, A., Connection formular for second-order differential equations with a complex parameter and having an arbitary number of turning points, Math. Nachr. 165 (1994), $205-229$.

17. Yurko, V.A., On integral transforms connected with differential operators having singularities inside the interval, Integral Transforms and Special Functions 5 (1997), no. $3-4,309-322$. 
18. Yurko, V.A., Boundary value problems with a parameter in the boundary conditions, Izv. Akad. Nauk Armyan. SSR, Ser. Mat., 19 (1984), no. 5, 398 - 409; English transl. in Soviet J. Contemporary Math. Anal., 19 (1984), no. 5, 62 - 73.

19. Yurko, V.A., An inverse problem for second order differential operators with regular boundary conditions, Math. Zametki 18 (1975), no 4, 569 - 576; English transl. in Math. Notes 18 (1975), no. 3 - 4, 928 - 932. 